\title{
Intuitive, Localized Analysis of Shape Variability
}

\author{
Paul Yushkevich, Stephen M. Pizer, Sarang Joshi, and J. S. Marron \\ Medical Image Display and Analysis Group, \\ University of North Carolina at Chapel Hill.
}

\begin{abstract}
Analysis of shape variability is important for diagnostic classification and understanding of biological processes. We present a novel shape analysis approach based on a multiscale medial representation. Our method examines shape variability in separate categories, such as global variability in the coarse-scale shape description and localized variability in the fine-scale description. The method can distinguish between variability in growing and bending. When used for diagnostic classification, the method indicates what shape change accounts for the discrimination and where on the object the change occurs. We illustrate the approach by analysis of $2 \mathrm{D}$ clinical corpus callosum shape and discrimination of simulated corpora callosa.
\end{abstract}

\section{Introduction}

Analysis of shape has begun to emerge as a useful area of medical image processing because it has the potential to improve the accuracy of medical diagnosis, the correctness of image segmentation, and the understanding of processes behind growth and disease. We present a novel 2D shape analysis method that can describe shape variability in intuitive terms, and pinpoint the places where variability is most pronounced. We use our method to analyze the shape of the mid-sagittal slice of the corpus callosum.

For example, consider the shapes in Fig. 1 which shows characteristic representatives of three classes of shapes. Our method can detect that the three classes are different. It can show that there is a global difference in width and bending between classes 1 and 2, and that near the middle of the object there is a local difference between classes 1 and 3 .

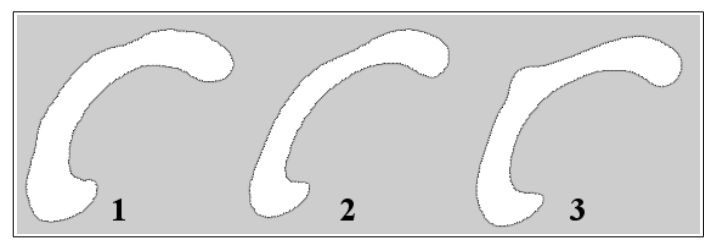

Fig. 1. Representatives of three classes of shapes whose differences can be described globally and locally. 
Methods in 2D shape analysis can typically be divided into three high level steps. First, a geometric representation is established. Second, a set of features is derived from the representation; these features must be invariant under the similarity transform. Finally, a statistical analysis method is chosen and applied to the features. The shape analysis literature can be categorized by the decisions taken at each step.

In their seminal paper on shape analysis, Cootes et al. represent shapes using a point boundary model, which is a list of coordinates of points on the object boundary. Invariance under rigid transform is achieved by alignment via the Procrustes algorithm; aligned boundary positions form the features. Principal component analysis (PCA) is used to gain both a qualitative and a quantitative description of global shape variability [2].

Both Staib \& Duncan and Szekely et al. represent boundaries in 2D as a weighted sum of Fourier basis functions and perform statistical analysis on the weights $[8,9]$. In both methods the representation inherently provides invariance under the similarity transform.

Bookstein and others use biological landmarks to represent shapes [3]. Landmarks are aligned by the Procrustes algorithm. Analysis is based upon thin plate spline warps which map one set of landmarks into another.

In a study of corpora callosa, Golland et al. represent 2D objects using a fixed topology skeleton, which is a snake-like approximation to the medial axis of an object [5]. Width and approximate curvature are sampled along the skeleton and serve as features. These features are inherently invariant under similarity transform. Classification is performed using linear discrimination and support vectors.

Our method also uses the same three step framework. We make the following decisions at each step. We describe shapes using a multiscale medial representation. A set of features, similar to those by Golland [5], is derived from the representation; the features are invariant under the similarity transform. We classify shapes using Fisher linear discriminants.

Our method is unique because it focuses on dividing the description of the shape variability into parts. We can analyze variability in the coarse-scale description of entire object separately from the fine-scale variability in a part of the object. The method also allows separate examination of growth-type shape changes, such as narrowing and elongation, and bending-type shape changes. Our choice of representation makes these two types of separability possible.

We describe objects using m-reps, which are formally defined by Pizer et al. as a discrete multiscale medial representation of shape [6]. M-reps capture shape in intuitive terms, such as widening, bending, and elongation because they are medial. According to Blum, whose medial axis work lead to the development of m-reps, the medial description is especially suitable for biological objects [1].

We say that m-reps are multiscale because they have an inherent level of boundary tolerance. A coarse-scale m-rep describes the general properties of shape, paying little attention to the details of the boundary. A fine-scale $\mathrm{m}$ rep captures detailed shape properties. Both types of $\mathrm{m}$-reps provide different 
information about shape, and a rich description is obtained when m-reps at different levels of detail are used together.

To discriminate between classes of objects based on their shape, we apply existing classification methods at multiple scales and locations. For example, in a simulated set of corpora callosa, which Fig. 1 illustrates, we find that discriminability between classes 1 and 3 is strongest at the bump location.

\section{Methods}

Fig. 2 summarizes our localized shape discrimination method. As most shape classifiers, ours is trained on a sample set of shapes extracted from images. Presently these are binary images of the corpus callosum.

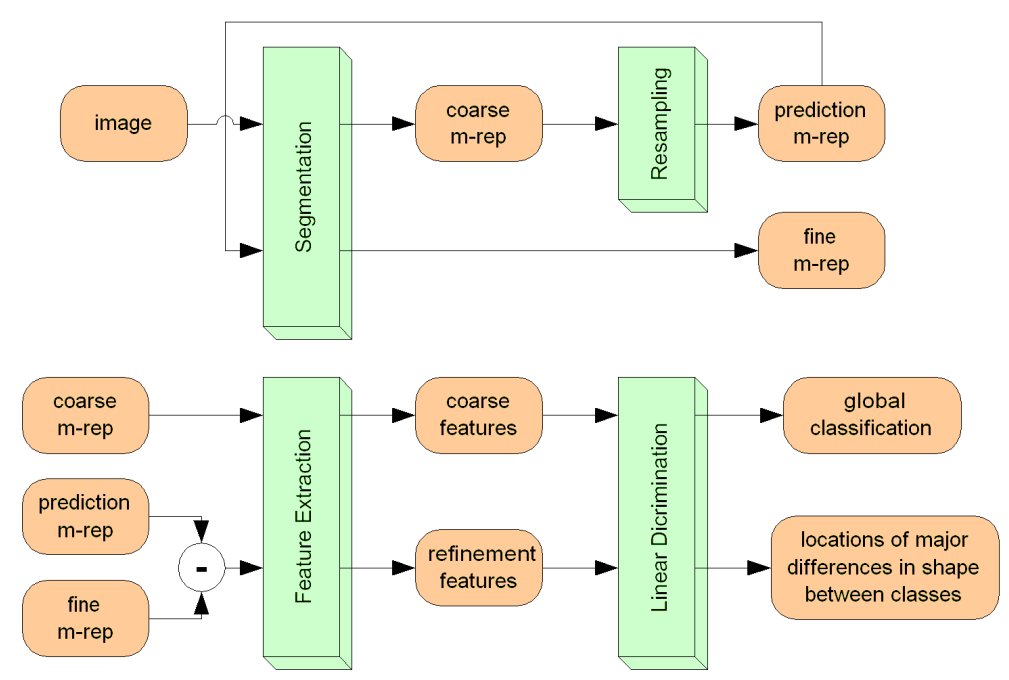

Fig. 2. The components and flow of the localized shape discrimination method. Shape features are extracted from each input image.

Our method analyzes shape at multiple levels of detail. Each shape is represented by both a coarse scale and a fine scale model. M-reps, defined in [6] serve as the shape representation because they incorporate scale-sensitive metrics and provide a geometrically rich shape description. A pair of m-reps, one with five medial atoms and a large boundary tolerance and another with nine atoms and a smaller tolerance are fitted to each image; these m-reps are called the coarse and the fine m-reps (Fig. 3).

The coarse m-rep is computed first by warping a template five-atom m-rep to maximize image match along its implied boundary. Image match is computed 

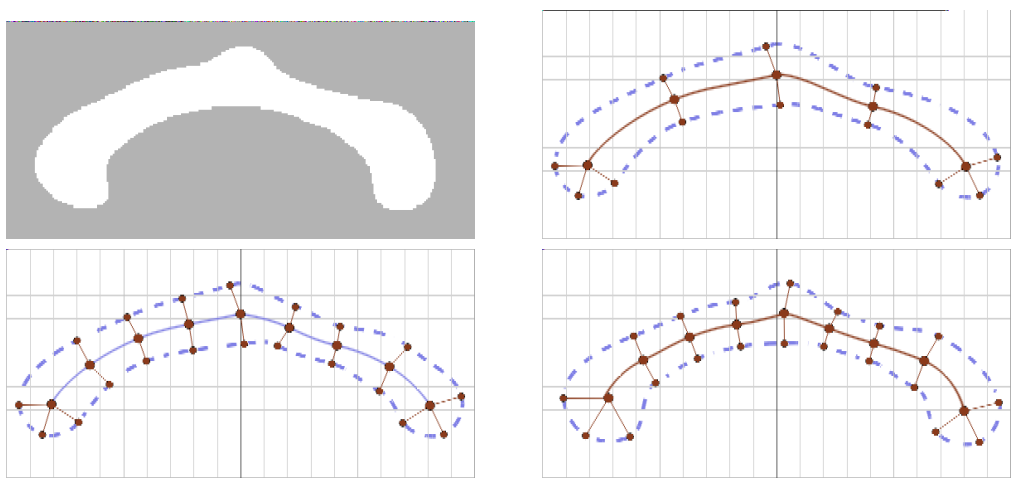

Fig. 3. A typical simulated corpus callosum image (top left), a coarse m-rep (top right), a prediction m-rep (bottom left), and a fine m-rep (bottom right).

using a Gaussian derivative operator with aperture proportional to local width of the m-rep; the constant of proportionality is set large $(\rho=1.0)$ for coarse m-reps. Medial atoms are constrained to remain at equal distances from each other during warping.

Using a medial interpolation technique outlined in [10] we resample the coarse m-rep, inserting a new medial atom half-way between each pair of existing atoms to form a prediction m-rep. The latter has the same implied boundary as the coarse m-rep but 9 atoms instead of 5 . The prediction $\mathrm{m}$-rep is again warped to fit the image, this time using a smaller aperture-to-width ratio $\rho=0.5$.

The three m-reps computed for each input image are used to derive statistical features. These features are geometrical in nature and describe shape properties such as growth and bending; these features are invariant under similarity transform. Two sets of features are computed. From the coarse m-rep we derive coarse features which describe relationships between neighboring medial atoms. From fine and prediction m-reps we derive the refinement features which measure geometrical differences between corresponding pairs of medial atoms. Refinement features describe the residual information gained from measuring shape at a smaller scale.

We use coarse features to discriminate between classes of shapes based on global shape properties. We perform three types of global discrimination, one based on just the bending features, one based on just the growth features, and one on the whole set of global features. When we compare the strengths of the three discriminations, we learn whether the differences between the classes are characterized more by differences in bending or growth.

We use refinement features to find locations on the shape where differences between classes are most profound. We perform a separate dicrimination based on the refinement features of each of the 9 atoms present in the fine m-rep. By comparing the relative strengths of the discriminations we find the locations on the object where the two classes differ most significantly. 
For each feature set, discrimination between two classes is performed by first reducing the features to one dimension by projection on the Fisher linear discriminant and then performing the Student $t$ test. [4]. The $p$-value of this test indicates the separability strength between the two classes.
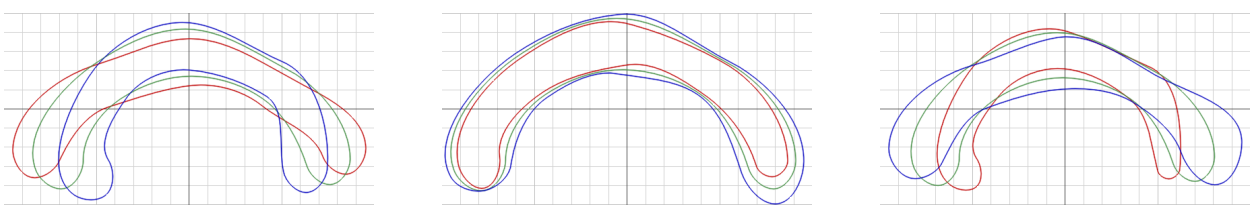

Fig. 4. Primary mode of variability in bending features (left), growth features (middle), and combined growth and bending features (right) in a class of corpus callosum shapes. Displayed are implied boundaries of m-reps corresponding to points at $-2,0$, and +2 standard deviations from the mean along the primary mode.

Additionally, we compute the primary modes of shape variability in each class or whole population, following a technique similar to Cootes et al. [2]. The feature extraction step is invertible, allowing reconstruction of m-reps from points in feature space, and hence modes of variability can be visualized as animations. We can analyze and visualize shape variability separately in terms of growth and bending (Fig. 4).

\section{Experimental Results}

We demonstrate the diagnostic ability of our method in a case that supports discrimination by constructing three artificial classes of objects based on the corpus callosum shape with representatives shown in Fig. 1. Classes 1 and 2 differ slightly in coarse shape while classes 1 and 3 have same basic coarse shape but differ locally because class 3 has a random bump at the midbody. Our hypothesis is that the method would be able to discriminate between classes 1 and 2 globally while discriminating locally between classes 1 and 3 .

Our simulation is based on elliptical harmonic representation of the segmented corpora callosa, kindly provided by the group headed by Guido Gerig [9]. The flexibility of the harmonics representation allows one to easily generate artificial shapes that resemble the corpus callosum. We create two Gaussian distributions in the PCA space of the spherical harmonics. These have different means and the same covariance. We take a random sample of 25 points from each distribution; each point corresponds to a corpus callosum shape that is rasterized. Thus we obtain training images for simulated classes 1 and 2 .

The third simulated class is sampled from the same distribution as class 1 but each shape in this class has an artificial bump. To create this class, we follow the same procedure as for class 1 , but before rasterization we add a perturbation 
in the shape of a truncated cosine function to the boundary. The location and amplitude of the perturbation follow the normal distribution.

\begin{tabular}{|l|c|c|c|c|c|c|c|c|c|}
\hline & 1 & 2 & 3 & 4 & 5 & 6 & 7 & 8 & 9 \\
\hline $\mathbf{1}$ vs $\mathbf{2}$ & -1.63 & -3.49 & -1.00 & -1.62 & -2.64 & -3.75 & -2.82 & -2.72 & -6.80 \\
\hline $\mathbf{1}$ vs 3 & -1.77 & -1.26 & -4.83 & -9.72 & -8.86 & -9.23 & -0.83 & -1.25 & -2.20 \\
\hline
\end{tabular}

Table 1. Decimal exponents of $p$-values from Student $t$-test that show separability between class pairs 1 vs. 2 and 1 vs. 3 for nine sets of refinement features.

We use leave-one-out analysis to test the classification ability of our method. Using coarse features, we can discriminate between classes 1 and 2 with $70 \%$ accuracy. This result is encouraging because the corresponding classes in spherical harmonics coefficient space have $80 \%$ discrimination accuracy.

Table 1 demonstrates our ability to locate the bump. Here discrimination between classes was performed on the refinement features at each of the medial atoms. For classes 1 and 3, the $p$-values for atoms near the middle of the figure are much smaller than at the ends, indicating stronger separability. Contrast with the same discriminations for classes 1 and 2 . The strongest separability is found at one of the ends.

\section{Discussion and Conclusions}

The major contribution of this paper is the development of a shape analysis method that leverages the intuitive and multi-scale properties of the medial representation. We demonstrate this technique by the analysis of simulated data. The application to real data remains.

Our statistical features have distributions that qualitatively do not appear non-Gaussian, rather distributions of some features have outliers and are multimodal. Further examination is needed to improve the normality of the features. Statistical methods that do not assume normality may also improve analysis.

We are extending the method to three dimensions because most of the potential medical applications deal with 3D images of human anatomy. Transition to $3 \mathrm{D}$ is possible in practice because recent progress in medial segmentation lets us extract m-reps of 3D anatomical structures semi-automatically [7]. M-rep interpolation and resampling pose the major theoretical difficulty. We plan use the method to analyse hippocampal shape in alzheimers disease.

To be useful in practice, our method can not be limited to single figure objects. Few shapes can be accurately represented by a single chain of medial atoms (or a single mesh in $3 \mathrm{D}$ ). The capability to analyze multi-figural objects can be achieved easily if the medial branching topology is the same for all shapes in the training set. In this case, we must simply add new features that describe figure-to-figure relationships. 
Homology is a problem common to all extant boundary and medial based shape analysis approaches, including ours. We establish homology by sampling medial atoms at equal spacing between the ends of the medial axis. Such correspondence is too sensitive to the placement of the ends. Establishment of homology based on the training statistics requires considerable research effort.

\section{Acknowledgements}

This work would not have been possible without the invaluable advice and support from many MIDAG members, most notably, Stephen Aylward, Daniel Fritsch, Guido Gerig, Sean Ho, Martin Styner, and Andrew Thall. We thank Guido Gerig and Sean Ho for provision of corpus callosum data and harmonic analysis methods. We are grateful to Yonatan Fridman and Gregg Tracton for aid in m-rep segmentation. This work was carried out under the partial support of NIH grants P01 CA47982 and R01 CA67183. Some of the equipment was provided under a gift from the Intel Corporation.

\section{References}

1. H. Blum, A transformation for extracting new descriptors of shape, Models for the Perception of Speech and Visual Form, MIT Press, 1967.

2. T. Cootes, C. Taylor, D. Cooper, and J. Graham, Active shape models - their training and application, Computer Vision, Graphics, and Image Processing: Image Understanding 1 (1994), no. 61, 38-59.

3. I. Dryden and K. Mardia, Statistical shape analysis, John Wiley \& Sons, New York, 1998.

4. R. Duda and P. Hart, Pattern classification and scene analysis, John Wiley \& Sons, New York, 1973.

5. P. Golland, W.E.L. Grimson, and R. Kikinis, Statistical shape analysis using fixed topology skeletons: Corpus callosum study, International Conference on Information Processing in Medical Imaging, LNCS 1613, Springer Verlag, 1999, pp. 382-388.

6. S. Pizer, D. Fritsch, P. Yushkevich, V. Johnson, and E. Chaney, Segmentation, registration, and measurement of shape variation via image object shape, IEEE Transactions on Medical Imaging 18 (1999), 851-865.

7. S.M. Pizer, T. Fletcher, Y. Fridman, D.S. Fritsch, A.G. Gash, J.M. Glotzer, S. Joshi, A. Thall, G Tracton, P. Yushkevich, and E.L. Chaney, Deformable m-reps for $3 d$ medical image segmentation, In Review, ftp://ftp.cs.unc.edu/pub/users/nicole/defmrep3d.final.pdf, 2000.

8. L.H. Staib and J.S. Duncan, Boundary finding with parametrically deformable models, IEEE Transactions on Pattern Analysis and Machine Intelligence 14 (1992), no. 11, 1061-1075.

9. G. Székely, A. Kelemen, Ch. Brechbühler, and G. Gerig, Segmentation of 2-D and 3-D objects from MRI volume data using constrained elastic deformations of flexible Fourier contour and surface models, Medical Image Analysis 1 (1996), no. 1, 19-34.

10. P. Yushkevich, Pizer S.M., S. Joshi, and Marron J.S., Intuitive, localized analysis of shape variability, UNC Dept of Computer Science Technical Report. http://www.cs.unc.edu/ pauly/ipmi2001/ipmi2001.pdf, 2001. 